\title{
Cardiovascular modeling of congenital heart disease based on neonatal echocardiographic images.
}

\section{$\operatorname{AUTHOR}(\mathrm{S}):$}

Nakao, Megumi; Maeda, Kazuma; Haraguchi, Ryo; Kurosaki, Ken-ichi; Kagisaki, Koji; Shiraishi, Isao; Nakazawa, Kazuo; Minato, Kotaro

\section{CITATION:}

Nakao, Megumi ...[et al]. Cardiovascular modeling of congenital heart disease based on neonatal echocardiographic images.. IEEE transactions on information technology in biomedicine 2012, 16(1): 70-79

\section{ISSUE DATE:}

2012-01

URL:

http://hdl.handle.net/2433/154586

\section{RIGHT:}

(c) 2012 IEEE. Personal use of this material is permitted. Permission from IEEE must be obtained for all other uses, in any current or future media, including reprinting/republishing this material for advertising or promotional purposes, creating new collective works, for resale or redistribution to servers or lists, or reuse of any copyrighted component of this work in other works.; この論文は出版社版でありません。引用の際には出版社版をご確認ご利用ください。; This is not the published version. Please cite only the published version. 


\title{
Cardiovascular Modeling of Congenital Heart Disease Based on Neonatal Echocardiographic Images
}

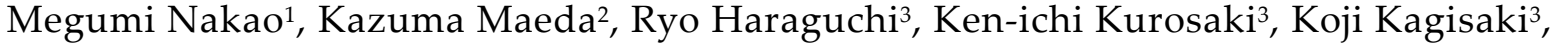 \\ Isao Shiraishi ${ }^{3}$, Kazuo Nakazawa ${ }^{3}$ and Kotaro Minato ${ }^{2}$ \\ ${ }^{1}$ Graduate School of Informatics, Kyoto University \\ ${ }^{2}$ Graduate School of Information Science, Nara Institute of Science and Technology \\ ${ }^{3}$ National Cerebral and Cardiovascular Center
}

\begin{abstract}
This paper proposes a three-dimensional cardiovascular modeling system based on neonatal echocardiographic images. With the system, medical doctors can interactively construct patient-specific cardiovascular models, and share the complex topology and the shape information. For the construction of cardiovascular models with a variety of congenital heart diseases, we propose a set of algorithms and interface that enable editing of the topology and shape of the three-dimensional models. In order to facilitate interactivity, the centerline and radius of the vessels are used to edit the surface of the heart vessels. This forms a skeleton where the centerlines of blood vessel serve as the nodes and edges, while the radius of the blood vessel is given as an attribute value to each node. Moreover, parentchild relationships are given to each skeleton. They are expressed as the directed acyclic graph (DAG), where the skeletons are viewed as graph nodes and the connecting points are graph edges. The cardiovascular models generated from some patient data confirmed that the developed technique is capable of constructing cardiovascular disease models in a tolerable timeframe. It is successful in representing the important structures of the patient-specific heart vessels for better understanding in preoperative planning and electric medical recording of the congenital heart disease.
\end{abstract}

Keywords - Cardiovascular modeling, interactive editing, congenital heart disease, echocardiography, telediagnosis

\section{INTRODUCTION}

Echocardiography plays a major role in the diagnosis of heart disease and in a versatile understanding of the patient's condition $[1,2]$. As the patient is not exposed to radiation, the imaging procedure can be repeatedly performed. Immediate observations are possible at the bedside due to its real-time imaging performance. A functional as well as morphological diagnosis is allowed based on echocardiography images. Accordingly, echocardiography is the firstchoice for the diagnosis of neonatal congenital heart disease. Many advanced medical centers that treat congenital heart disease only use echocardiography during surgical proce- dures. The precipitous change in neonatal hemodynamics when the blood circulation reverses at birth can result in a sudden change in the condition of a subject with congenital heart disease, so early diagnosis is important. There are growing expectations for telediagnostic techniques to address the lack of specialists capable of making such a diagnosis.

Various attempts have been made at telediagnosis using echocardiography, but it can be difficult for the sonographer and the diagnostic doctor to share information on the probe position and angle and the image range. At the National Cerebral and Cardiovascular Center (NCCC), neonates with suspected congenital heart disease are subjected to tele-echocardiography using a horizontal sequence scanning method [3]. With this method, the probe is constantly applied perpendicular to the body axis during scanning imaging. The sonographer and the diagnostic doctor therefore know how the probe is being operated before the procedure. An experienced doctor would be capable of making a diagnosis from the echocardiogram images received, based on a mental image of the three-dimensional structure of the heart. However, the expertise and experience are needed to understand the three-dimensional cardiovascular structure from the echocardiogram images. It would be extremely difficult for most of medical staffs involved in the treatment, such as the surgeon performing the operation, to gain an accurate understanding of the patient's condition from the echocardiogram images. At present, cardiovascular specialists brief surgeons and other staffs on the patient's condition using two-dimensional images or diagrams (Figure 1). However, there is no efficient way to communicate the condition of an individual patient with congenital heart disease and it is difficult to gain a completely shared understanding.

The patient's QOL (Quality of Life) can be improved if specialists and other staffs share a common understanding of the patient's condition. When analyzing congenital heart disease, it is particularly important to understand the shape of the blood vessels and their relative positional relationships. Specialists, surgeons and other staffs need to share information on the cardiovascular morphology. Therefore, three-dimensional cardiovascular models specific to echo- 


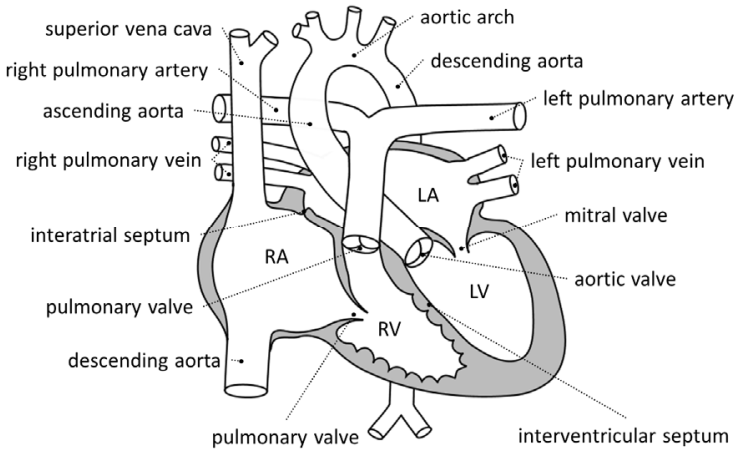

Figure 1. Two-dimensional illustration of a normal heart.

cardiogram images of patients is useful for various situations such as preoperative planning, telediagnosis and preparing electronic medical records.

Researchers have recently proposed a three-dimensional echocardiography approach whereby multiple echocardiogram images are used to reconstruct a three-dimensional echocardiogram image $[4,5,6,7]$. However, at present, direct visualization of the images does not provide sufficient image quality needed for a diagnosis of complex congenital heart diseases. Much research is underway into the automatic extraction of regions of interest (ROI) from multiple two-dimensional echocardiography images $[2,8,9,10]$. This segmentation and reconstruction approach has been well explored in medical imaging literature [11, 12, 13]. However, as the echocardiogram images lack required resolution or the whole shape of the specific organs, the modeling results are not necessarily very accurate. Each frame of echocardiographic images obtained using horizontal sequence scanning often provides incomplete information of anatomical structures that only an expert specialist could know. Therefore, it is extremely difficult to build a cardiovascular model only from echocardiographic images. This feature can severely limit the applicability of segmentation-based approaches that have been developed for reconstruction from CT/MRI volume data $[14,15,16]$.

In this study, we present a cardiovascular modeling system not only using echocardiogram images but with the simple interaction to the model by the user. We mainly focus on the expression in a three-dimensional format of the disease state as seen in a mental image by a cardiovascular specialist. Therefore, the goal of this system is to construct a cardiovascular model that expresses the feature of the congenital heart disease as diagnosed by the specialist. When creating patient-specific cardiovascular models, specialists refer to the echocardiographic images and interactively edit cardiovascular models prepared in advance. In order to express the extremely diverse and complex cardiovascular morphology seen in congenital heart disease, we categorize cardiovascular morphological characteristics into shape and topology, and propose methods to interactively edit these categories. We provide a shape-editing interface to enable direct input onto the echocardiographic images for accurately capturing the patient's individual cardiovascular morphology. Also, we introduce topology-editing interface to easily create a cardiovascular model that incorporates the (a)

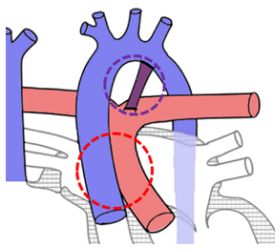

(c)

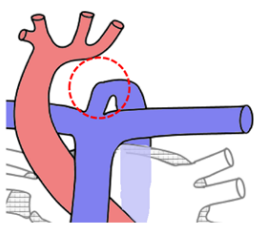

(d)

(b)
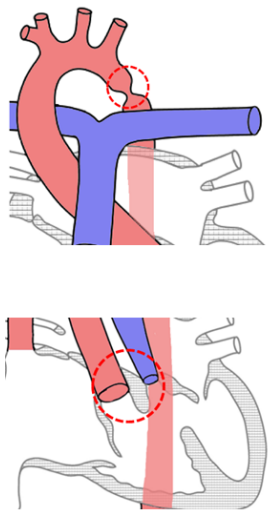

Figure 2. Examples of congenital heart disease: (a) complete transposition of arteries, (b) coarctation of the aorta, (c) interruption of the aortic arch and (d) double outlet right ventricle.

complexity of the system. Through several tests with medical staffs, we confirm that our approach can efficiently express the key features of the congenital heart disease from echocardiographic images.

\section{CARDiovasculaR MODEL DATA STRUCTURES AND EDITING ALGORITHMS}

\subsection{Congenital heart disease}

Congenital heart disease involves some abnormality in the blood vessel structures connecting to the heart or in the heart itself that has been present since birth. The condition occurs in approximately 8 of every 1,000 neonates. There are many types of congenital heart disease and the situation is further complicated by the parallel use of multiple disease names. It takes several years of specialist training to be able to surmise the patient's condition from the disease name alone. Even within the same disease name, a change in the patient's condition can result in completely different symptoms and treatment methods.

Figure 2 shows examples of congenital heart disease targeted in this research. Complete transposition of the great arteries (TGA) (Figure 2a) is where the aorta and pulmonary arteries are transposed, with the aorta originating from the anatomical right ventricle (RV) and the pulmonary artery from the anatomical left ventricle (LV). Because of this, the aorta is located forward of the pulmonary artery or even forward and to the right. As the pulmonary circulatory and systemic circulatory systems are not connected, there must be a ventricular septal defect (VSD), atrial septal defect (ASD), or patent ductus arteriosus (PDA) for the subject to survive. In coarctation of the aorta (Figure $2 b$ ), there is a coarctation where the ductus arteriosis flows into the descending aorta or where the ligamentum arteriosum attaches. The degree of coarctation can range from extremely minor to almost complete closure. With an interrupted aortic arch (IAA) (Figure 2c), there is extreme coarctation of the aorta such that some part of the aortic arch is completely closed or missing. In many cases, IAA is concomitant with 
VSD or PDA. Marked hypoplasia is usually observed in the aortic arch between the ascending aorta and the interrupted section. Double-outlet right ventricle (DORV) (Figure 2d) generally refers to a disease where both the aorta and the pulmonary artery originate from the anatomical RV. The condition is commonly defined as a disease where $150 \%$ or more of the great arteries originate from the RV. Around $65 \%$ of DORV cases exhibit a parallel relationship between the aorta and pulmonary artery and this is the most common type of DORV.

It is clear, therefore, that congenital heart disease is an extremely diverse and complex condition and that the cardiovascular morphology of this condition should be characterized using two different categories. The first is cardiovascular shape as defined by the centerline and radius of the blood vessels. The second is topology, which comprises the number of blood vessels and their connective interrelationships. By editing the shape and the topology of the cardiovascular model, we can express the cardiovascular morphology specific to a particular patient.

\subsection{Cardiovascular model data structures}

In the field of computer graphics (CG), the most common method used to express a three-dimensional object is a triangle surface mesh that defines the object's surface. Recently published papers $[17,18]$ covered various mesh generation and editing methods. With conventional surface mesh models, it is difficult to find a way to handle the radius of the cardiovascular model and it is not easy to edit the topology because of the need to process detailed mesh divisions or detect conflicts. In this research, we define data structures as shown below to be able to interactively edit cardiovascular model shape and topology.

First, we define a cardiovascular centerline for a skeleton $S_{i}$ comprising nodes and edges (Figure 3a). We then apply a radius $r_{j}$ to each skeleton node $\mathbf{N}_{j}$ as an attribute value, allocate the top surfaces (Figure $3 b$ ), produce a surface mesh from the top surfaces at each node, and use this as the blood vessel surface. To define coordinates for each node, a plane perpendicular to the edge linking the node to its adjacent node (or linking the adjacent node and the end point as there is only one adjacent node at the end point) is extended outward from the top surface of the node. The surface nodes are distributed at fixed intervals along its circumference from the radius of each node. We define the connective relationships of the cardiovascular models according to the parent-child relationship between each cardiovascular model. However, this does not allow the expression of vascular rings structured like a tree. Therefore, we express the parent-child relationships as a directed acyclic graph (DAG) where each cardiovascular model is viewed as a graph node and the connecting points are graph edges. This enables the expression of cardiovascular morphology accompanying a vascular ring (Figure 4).

\subsection{Shape editing}

\section{A. Vessel center line}

When editing the centerline, we define the position of each node on the cardiovascular model skeleton as a con-

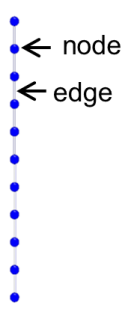

(a)

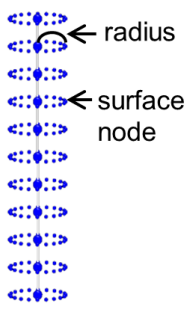

(b)
Figure 3 Data structure of cardiovascular model. (a) skeleton and (b) surface of a vessel model

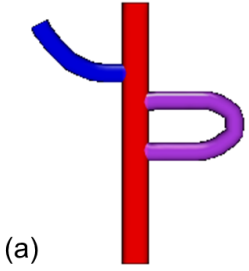

(b)

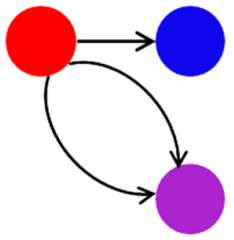

Figure 4 Directed acyclic graph representation of vessel topology: (a) an example of vessel topology and $(b)$ its direct acyclic graph

straint by user's input. In order to reduce the burden on the user, the user needs to be able to edit in real time, and with only a few operations, the nonlinear changes seen in vessel shapes, such as twists and bends. Therefore for the centerline editing, user's interaction are limited to defining the position of some skeleton nodes. The model surfaces are smoothly modified in real time while node positions satisfy user's constraints.

In order to fulfill these conditions, we apply interactive surface editing method $[19,20]$ proposed by Nealen et al. to the skeleton editing. Figure 5 shows examples of the centerline editing. The manipulated points are shown in green and the fixed points in red. All nodes are free points before the user enters modifications. When node $\mathbf{N}_{j}$ on skeleton $S_{i}$ is manipulated, if there are no fixed points on the skeleton $S_{i}$, then the entire skeleton $S_{i}$ undergoes a parallel shift in line with the distance moved by node $\mathbf{N}_{j}$ (Figure 5a). If there are fixed points on skeleton $S_{i}$, then skeleton $S_{i}$ changes in shape to meet the constraints on the position of all the fixed and manipulated points (Figure 5b). The local frames placed on each node are smoothly rotated while satisfying the constraints defined by the user. The positions of the nodes are interpolated based on the rotated local frames. (Figure 5c).

When node $\mathbf{N}_{j}$ on skeleton $S_{i}$ is manipulated, if there is a parent on skeleton $S_{i}$, the connecting point to the parent is defined as a fixed point and skeleton $S_{i}$ changes in shape. At this time, the parent on skeleton $S_{i}$ does not change. If there is a child on skeleton $S_{i}$, the child changes in line with the changes in the skeleton $S_{i}$ as a constraint on the position of the connecting point with skeleton $S_{i}$. This process is repeated recursively to that child. If there are multiple children on skeleton $S_{i}$, the children are located using a breadth-first search (BFS) and unvisited children are changed. Such changes need to be done according to their sequential order for all parent-child relationships, with par- 
(a)

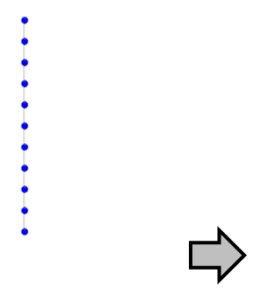

(b)

:

(c)

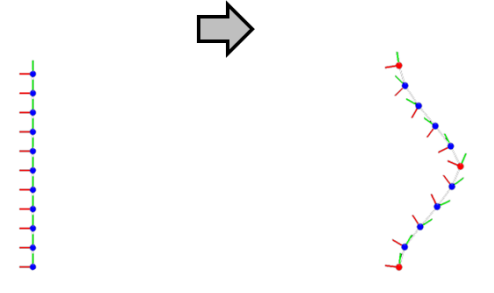

Figure 5. Centerline editing examples. (a) positional update with no fixed points, (b) shape update with fixed points and (c) skeleton deformation with local frames placed on each node

ents being visited before their children.

The example in Figure 6 shows manipulation of the red skeleton node. From the BFS, the blue, green, and purple children of the red node are visited before the orange. However, the blue node is the parent of green, so green must not be visited before blue. Moreover, purple is the parent of blue, so blue must not be visited before purple. In order to satisfy these sequential relationships, a topological sort is performed to identify the satisfactory sort order. Figure $6 \mathrm{c}$ shows the results when a topological sort is performed on Figure $6 \mathrm{~b}$. The search sequence is therefore red $>$ purple $>$ blue $>$ green $>$ orange.

\section{B. Vessel radius}

When editing the radius, we define the radius of each node on the cardiovascular model skeleton as a constraint on user-entered modifications. With radius editing, we need to be able to express, with a only few operations, the shape of blood vessels that have different thicknesses all over as in hypoplasia and of blood vessels that have different thicknesses in a specific location as in coarctation. We therefore decided that the conditions when editing radii should meet the user-entered radial constraints and should be interpolated uniformly from the neighboring radius. In order to satisfy these conditions, this research defined the evaluation function described in the equation (1)

$$
\underset{r}{\arg \min }\left(\sum_{i, j \in \mathbf{E}}\left\|r_{i}-r_{j}\right\|^{2}+\sum_{i \in \mathbf{C}}\left\|r_{i}-r_{i}\right\|^{2}\right)
$$

Here, $r_{j}$ is the radius of node number $j, r_{j}^{\prime}$ is the constraint on the radius of node number $i, \mathbf{E}$ is the edge set, and $\mathbf{C}$ is the constraint set. The first term of this equation is to interpolate uniformly the neighboring radius, while the (a)

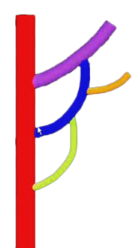

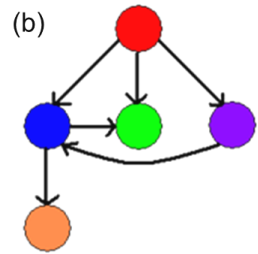

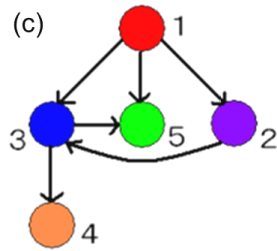

Figure 6 Topological sort of the skeletons to represent topology-based editing. (a) an example of the skeletons (b) directed acyclic graph representation and (c) sort result when manipulating red skeleton.

second term is to satisfy the constraints on the radius applied by the user.

Figure 7 shows examples of radius editing. Nodes where the radius has been manipulated are shown in green, while nodes with a fixed radius are shown in red. There are no radius constraints on any nodes before the user enters any changes. The radii of nodes manipulated by the user are set as fixed radii. When the radius $r_{j}$ of node $\mathbf{N}_{j}$ on skeleton $S_{i}$ is manipulated, if there are no constraints on the radii of any nodes on skeleton $S_{i}$, the radius of the entire skeleton $S_{i}$ becomes equal to that of radius $r_{j}$ (see Figure 7a). If there are constraints on the radii of nodes on skeleton $S_{i}$, then the radius of skeleton $S_{i}$ is edited to satisfy all the constraints on radii (Figure $7 \mathrm{~b}$ ).

\subsection{Topology editing}

To edit the topology of cardiovascular models, we propose four functions to enable additions, deletions, disconnection, and connections representing changes in connection points or connective relationships in the various cardiovascular models. With topology editing, we specify threedimensional positions within the cardiovascular model space by converting two-dimensional coordinates entered from mouse into three-dimensional coordinates. When the user specifies the two-dimensional coordinate $\mathbf{w}$ within the window, we obtain the depth value that corresponds to the two-dimensional coordinate $\mathbf{w}$. If there is a cardiovascular model in the view direction from the clicked point, we obtain the three-dimensional coordinate on the cardiovascular model surface that is at the forefront. If there is no cardiovascular model, we obtain a three-dimensional coordinate by applying a certain depth value. Using this method, we can create a cardiovascular model from the user-specified two-dimensional coordinate.

\section{A. Additions}

As a user enters strokes, we obtain three-dimensional coordinates while maintaining certain depth values at the stroke starting points. When stroke entry is completed, we distribute nodes at fixed intervals from the three-dimensional coordinates obtained and generate skeleton $S_{i}$. The number of nodes on skeleton $S_{i}$ is expressed as $n$, the endpoint on skeleton $S_{i}$ is $\mathbf{P}_{i}(i=0 \ldots n)$, each node on the existing skeleton is $\mathbf{N}_{j}(j=0 \ldots n)$, and the radius of node $\mathbf{N}_{j}$ is $r_{j}$. When there is a node $\mathbf{N}_{j}$ that satisfies the following equation for the endpoint $\mathbf{P}_{i}$ of skeleton $S_{i}$ : 
(a)
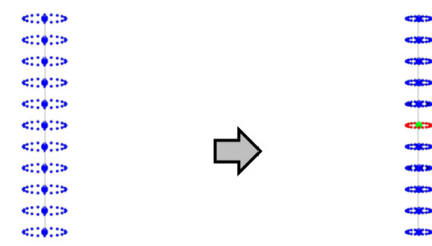

\section{$\underset{\infty \infty}{\infty}$}

(b)

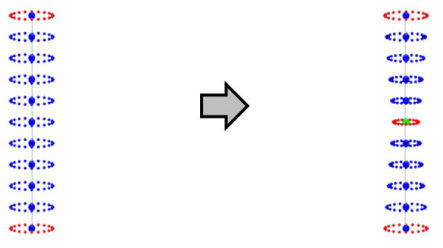

Figure 7. Radius editing examples. (a) radius update with no fixed points and (b) radius update with fixed points

$$
\left|\mathbf{P}_{i}-\mathbf{N}_{j}\right| \leq r_{j}
$$

we connect at the nearest node $\mathbf{N}_{c}$ from the endpoint $\mathbf{P}_{i}$ such that skeleton $S_{i}$ has a child with node $\mathbf{N}_{c}$.

\section{B. Deletions}

When there is a parent-child relationship on skeleton $S_{i}$ that has been selected by the user for deletion, the parentchild relationship of skeleton $S_{i}$ and the skeleton connected to skeleton $S_{i}$ are deleted. After deletion, there are no parent-child connections on skeleton $S_{i}$. Skeleton $S_{i}$ is then deleted.

\section{Disconnections}

While a user is entering strokes, we obtain depth values. If there is a cardiovascular model within the line of sight from the mouse pointer, we obtain an index from the node nearest to the mouse pointer. When stroke entry is completed, we divide skeleton $S_{i}$ with the nodes $\mathbf{N}_{0} \ldots \mathbf{N}_{n}$ that has nodes $\mathbf{N}_{i}$ corresponding to the lowest depth value of all the values obtained into two: skeleton $S_{i}^{\prime}$ with the nodes $\mathbf{N}_{0} \ldots \mathbf{N}_{i-1}$ and $S_{i}^{\prime \prime}$ with the nodes $\mathbf{N}_{i+1} \ldots \mathbf{N}_{n}$. When there is a skeleton that connects to node $\mathbf{N}_{i}$, we delete the parent-child relationship, and then delete node $\mathbf{N}_{i}$.

\section{Connections}

When a user selects and moves skeleton $S_{i}$, there is a change in the connection points and connective relationships between the selected skeleton and other skeletons. The same formula (2) as shown in the section on additions is used to determine the connection or disconnection of the selected skeleton with other skeletons.

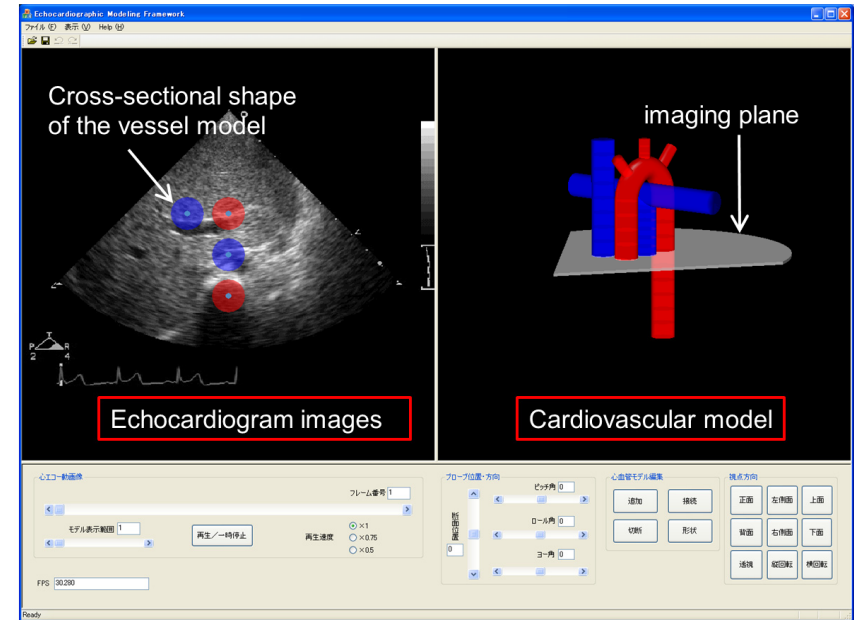

Figure 8. User interface of the cardiovascular modeling system.

\section{Cardiovascular Model Editing InTerface}

In this section, we introduce the composition of the proposed system and the cardiovascular model editing interface.

\subsection{System components}

We expect that the system proposed in this research will be used by specialists waiting for emergency transport to deliver an infant patient after tele-echocardiography by a specialist has confirmed the need for surgery. The specialists make a diagnosis after constructing a mental image of the three-dimensional structure of the disease state by building up echocardiographic images sent in real time from the remote site. Using the same process, we attempted to construct cardiovascular models with reference to echocardiographic images obtained from the telediagnosis. We also wanted to enable the entry of shape editing commands on top of the echocardiographic images. This process would not only allow the specification and manipulation of the model based on actually measured patient images, but also manipulation of two-dimensional images to enable the simple construction of complex cardiovascular models. Figure 8 shows an overview of the system proposed.

The system is composed of two screens, with the left screen displaying the echocardiographic images and the right screen the cardiovascular model. The system displays a plane (imaging position plane) on the cardiovascular model that shows where the echocardiographic images were recorded, while the cross-sectional shape of the cardiovascular model at the imaging position is superimposed on the echocardiographic images, enabling user entry directly onto the echocardiographic images. The cardiovascular model is categorized according to the type of blood flowing, with blood vessels transporting arterial blood colored red, blood vessels transporting venous blood colored blue, and blood vessels transporting a mix of blood colored purple. The user can switch blood vessel colors using a menu function. Undo and redo functionality is also possible. 

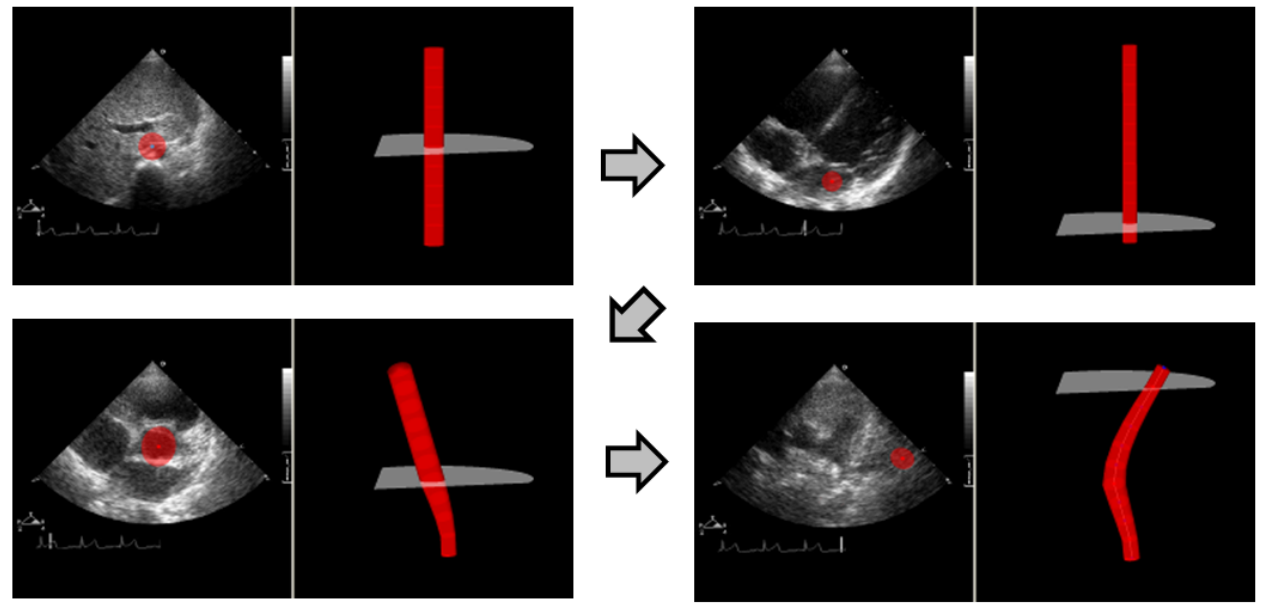

Figure 9 A flow of shape editing using echocardiogram images.
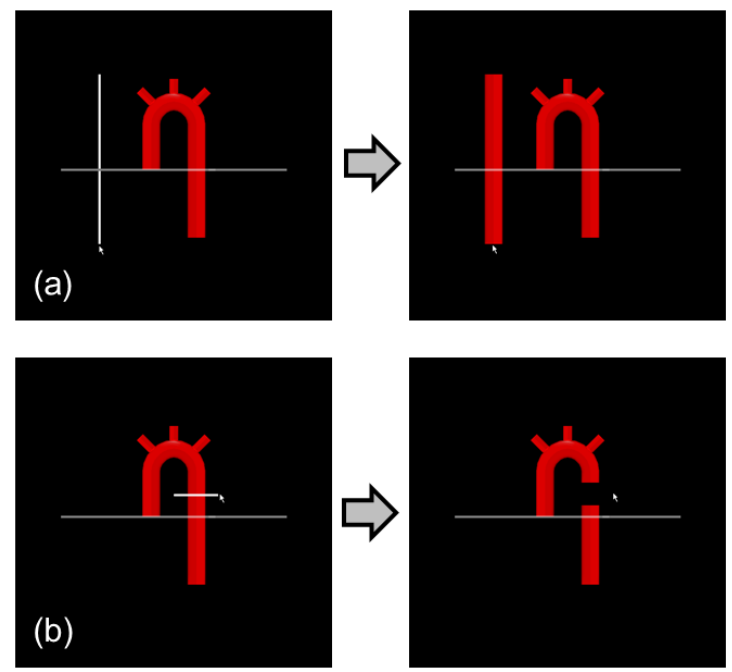

Figure 10 Topology editing examples (a) addition, (b) deletion, (c) cutting and (d) connection
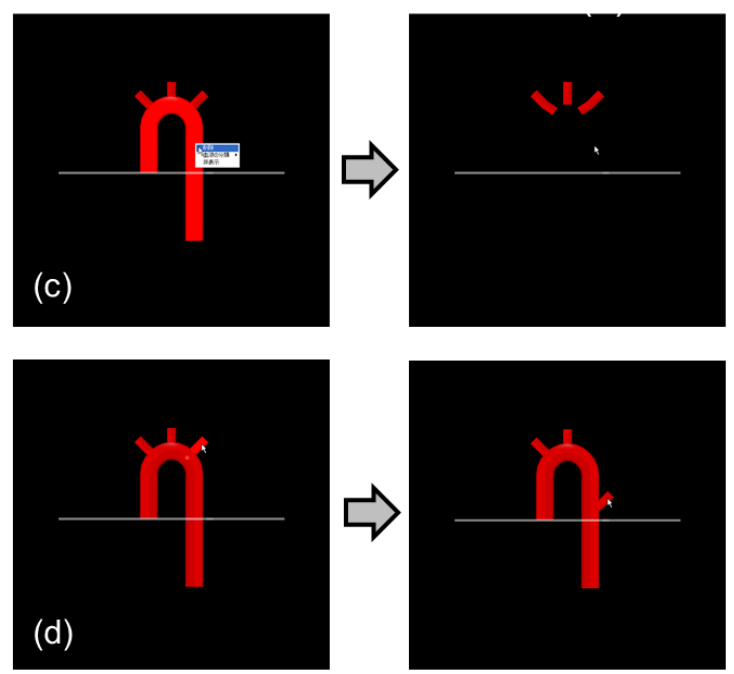

\subsection{Shape editing interface}

Shape editing of cardiovascular models is possible through two-dimensional data entry using the mouse on the system's left screen that displays the echocardiographic images. First, the user selects any image in the echocardiographic images by manipulating the slider in the control box positioned along the bottom. The user then specifies the imaging position plane for that image. The user left clicks to select the cardiovascular model superimposed on the echocardiographic image and can manipulate the position of the selected cardiovascular model within the image by dragging with the mouse. In the same way, the user left clicks to select the cardiovascular model superimposed on the echocardiographic image and can manipulate the radius of the selected cardiovascular model using the mouse scroll wheel. Through these user manipulations, the crosssectional shape of the superimposed cardiovascular model can be made to match the actual cardiovascular shape captured in the echocardiographic images. The user edits the cardiovascular shape by repeating this process for multiple cross-sectional positions. Figure 9 shows an example of shape editing flow. Also, see the supplemental movies.

\subsection{Topology editing interface}

Cardiovascular model topology can be edited by specifying three-dimensional positions within the cardiovascular model spaces based on two-dimensional data entry, for example by using the mouse on the system's right screen that displays the cardiovascular model. The user can toggle between additions, deletions, disconnections, and connections for topology editing using the button within the control box.

\section{A. Additions}

By entering strokes on the screen, the user adds new cardiovascular models with the stroke as the centerline (Figure 10a).

\section{B. Deletions}

A pop-up menu appears when the user right clicks on the cardiovascular model; the user selects Delete from this menu to delete a cardiovascular model (Figure 10b).

\section{B. Disconnections}

By entering strokes that cut across a cardiovascular model, 
the model can be disconnected at the intersection of the stroke and the model (Figure 10c).

\section{Connections}

The user can select by clicking on the cardiovascular model and move the entire selected cardiovascular skeleton in parallel by dragging with the mouse; if there is a node that meets the conditions described in section 2.4, a connection is made with the cardiovascular model possessing that node (Figure 10d).

\section{Results AND APPLICATIONS}

We implemented overall algorithms in $\mathrm{C}++$ and OpenGL in order to verify whether the proposed approach would allow specialists to construct patient - specific cardiovascular models from the echocardiographic images. Our interactive modeling system does not need any additional commercial software to create cardiovascular models. The experiment used an Intel Xeon X5450 $3.0 \mathrm{GHz}$ CPU and a standard PC with 3.25GB of memory, while the Intel Math Kernel Library was used for matrix operations. In this part of the research, we accompanied specialists and tested cardiovascular model structures using actual cases of a normal heart, complete TGA, and DORV obtained using a horizontal sequence scanning method.

Figure 11 shows the template model used in the experiment. The template model comprised seven skeletons, $S_{1}$ $S_{7}$ (Figure 11b), 226 skeleton nodes and 4520 points placed for the triangle mesh to represent the surface of the blood vessels. The model was constructed to be as close as possible to the cardiovascular morphology seen in the normal heart. The pulmonary vein is almost not imaged at all with the horizontal sequence scanning method and very few cases need to show pulmonary vein morphology for an understanding of the disease state. Therefore, the model built for the experiment targeted the aorta, pulmonary artery, and vena cava.

\subsection{Modeling results}

Figure 12a shows the results from cardiovascular modeling of a normal heart. The model took approximately 5 minutes to complete, with 17 manipulations (centerline: 13; radius: 3, additions: 1) and 4 cross sections manipulated. The aorta and pulmonary artery form a helical shape in the normal heart and the modeling results confirmed that this feature could be expressed. Figure 13 displays echocardiogram images and cross sections of the cardiovascular model on the four planes A, B, C and D depicted in Figure 12a. We note the cross sections include the branch and small vessel structures that cannot be extracted from the echocardiogram images. Since echocardiogram images do not include complete information of the anatomical structures, segmentation - based approaches have difficulty in representing detailed structures of the vessels. Our approach utilizes a deformable template to interpolate anatomical features common to sparsely selected images. The topology of the vessel model is customized and maintained during deformation. In the modeling step, the user only indicates the correct 2D position of the vessels based on the information clearly revealed on the reference echocardiogram images.
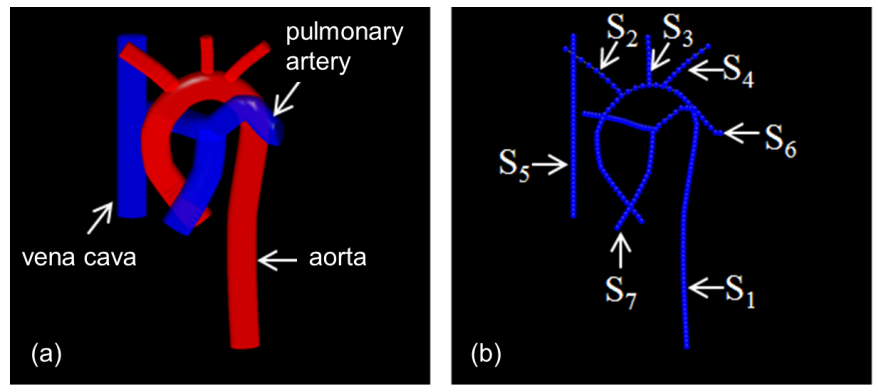

Figure 11 Cardiovascular template model created in our software, (a) surface representation and (b) its skeleton

However, our system can generate 3D cardiovascular models that satisfy user's knowledge from the partial or incomplete anatomical information given on the arbitrarily selected 2D planes.

Figure $12 \mathrm{~b}$ shows another result from modeling complete TGA. The model took approximately 7 minutes to complete, with 26 manipulations (centerline: 18; additions: 2; deletions: 3; disconnections: 3 ) and 5 cross sections manipulated The results showed that the model could show the aorta and pulmonary artery running parallel and a PDA connecting the aorta and pulmonary artery. The model also expressed the blood flow characteristics (arterial blood, venous blood) in the aorta and pulmonary artery. Finally, Figure $12 \mathrm{c}$ shows the results from modeling DORV. The model took approximately 7 minutes to complete, with 21 manipulations (centerline: 17; radius: 3; additions: 1 ) and 6 cross sections manipulated. The results confirmed that the model could express various characteristics, such as the aorta and pulmonary artery running parallel in the front.

We also tried to create the other complex blood vessel model to confirm the applicability of the developed modeling system. The target was the Circle of Willis [21], a circle of arteries that supply blood to the brain because it also has a unique, complex topology and shape. In this test, we started to draw all skeletons without any initial template model. As shown in Figure 14, we successfully modeled the key features of the Circle of Willis. The elapsed time for this modeling was around five minutes. The model also has the topological structure in our system, we can selectively edit the shape of the vessels. (see the supplemental movie).

\subsection{Evaluation with a Specialist}

In the tests, we asked the specialists on the usability and limitation of the developed system. Specialists used the developed system commented that the model could:

- Express patient-specific cardiovascular shapes and positional relationships that are important features when understanding the disease state

- $\quad$ Be directly used in a clinical setting

- Be applied when explaining to families or in electronic patient records

The specialists also suggested the following points for 


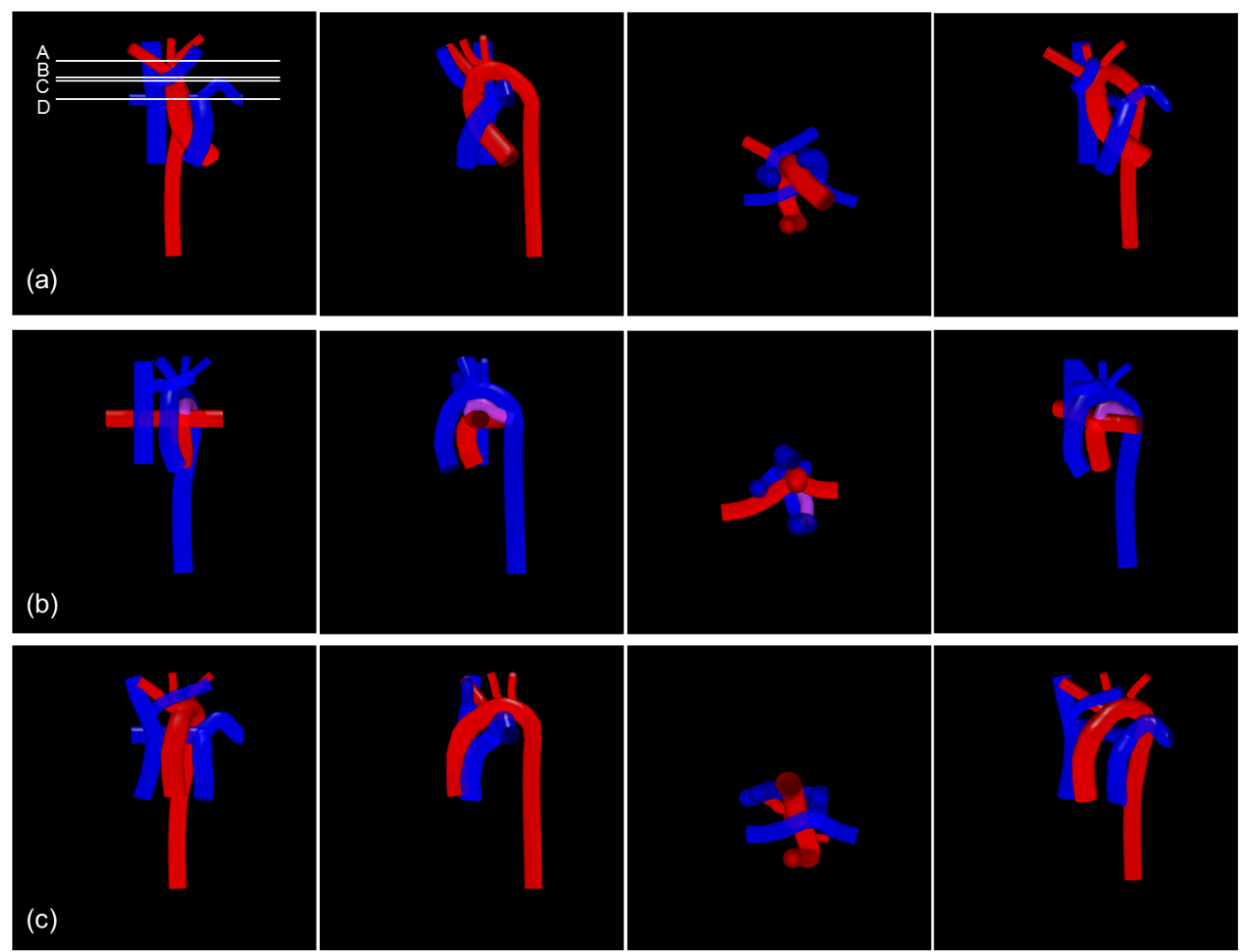

Figure 12 Cardiovascular modeling results from the template model with medical specialists. (a) normal heart, (b) complete transposition of great arteries and (c) double outlet ventricle
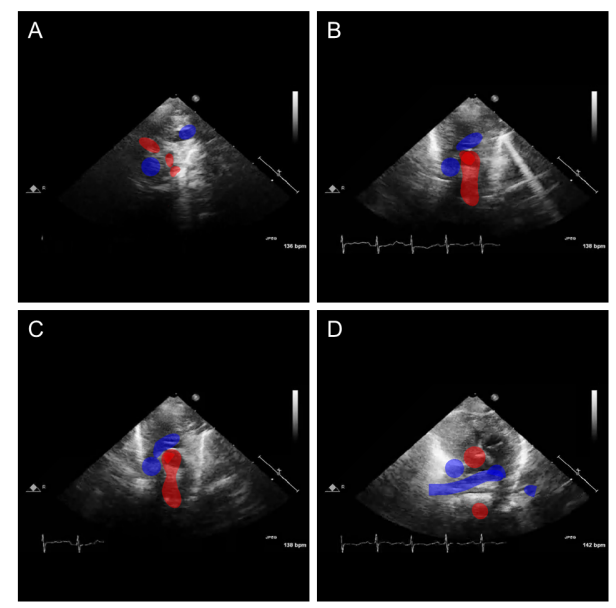

Figure 13 Cross sections of the interactively created cardiovascular model with the reference echocardiogram images.

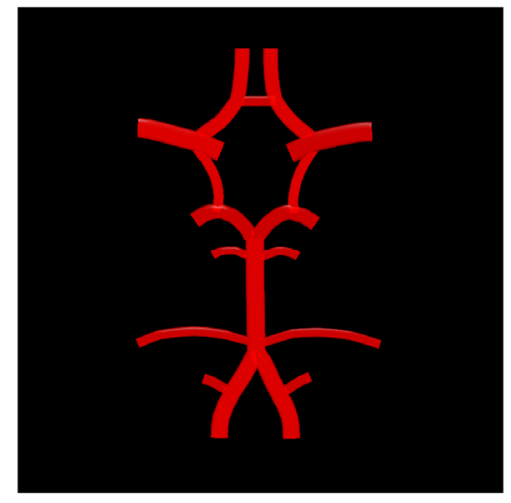

Figure 14 The Circle of Willis modeled on our system. The topological and geometrical features are successfully modeled.

further improvement:

- The disease state could be more easily understood if the system were designed to show the valve shape, ventricles, atria, pulmonary vein, and inferior vena cava

- Corrections need to be made if the probe positioning slips

\section{Discussion}

The several tests confirmed that the proposed method could be used to construct a cardiovascular model specific to a patient with congenital heart disease and to express features important in understanding the disease state. In 
this research, specialists unfamiliar with technical backgrounds such as mesh structures and modeling required less than 10 minutes to build models of three cases while receiving explanations of how to use the system. We think models could be built in shorter time frames once the specialists become proficient in the use of the system. As discussed in section 3.1, we assume that this system could be used while waiting for emergency transport carrying an infant patient to arrive.

With shape editing of the cardiovascular model, the results showed that the number of cross sections manipulated was $4-6$, while the number of manipulations was $17-26$. Therefore, the proposed method allows editing with only a few manipulations. The topology editing interface that enables data entry onto the echocardiographic image can be used to easily construct complex models, for example showing the positional relationship of the aorta and pulmonary artery crossing in a helical shape. This intuitive modeling environment is also applicable to other clinical situation such as daily surgical planning and medical education where medical staffs including young practitioners can share the anatomical knowledge or surgical procedures using tailor-made cardiovascular models.

In this research, rather than preparing a template for each disease state, we built cardiovascular models using the normal heart as a template in order to be able to adapt the model to various types of congenital heart disease. The results confirmed that it was possible to build models in a short timeframe using our proposed method, even for conditions where the shape differed significantly from the normal heart template.

As discussed in section 4.2, evaluation by the specialists highlighted the following issues to be addressed: how to model valve shape, ventricles, atria, the pulmonary vein, and the inferior vena cava and how to correct for slips in the probe position as there can be camera shake and the probe needs to be remain in close contact with the chest wall. These technical issues will be our future work.

\section{CONCLUSION}

This paper tries to address the difficulty of sharing a full understanding between specialists and other healthcare staff based on echocardiographic tele-diagnosis of congenital heart disease in neonates. The objective of the research was to express the congenital heart disease condition using echocardiographic images. We propose a system to construct patient-specific cardiovascular models whereby specialists interactively edit cardiovascular models prepared in advance while referring to echocardiographic images.

We propose an interface and methods to edit cardiovascular shape and topology in order to express the cardiovascular state in congenital heart disease, which encompasses a diverse range of conditions and can be extremely complex. To make the shape and topology editing interactive, we used assigned attribute values to a number of data structures on the cardiovascular model: the cardiovascular surface, the cardiovascular centerline of a skeleton comprising nodes and edges, and the cardiovascular radius of each skeleton node. We also used a DAG where skeletons are viewed as graph nodes and connecting points as graph edges.

Tests to verify the cardiovascular modeling using a number of actual cases showed that the system could be used to build a cardiovascular model based on echocardiographic images within a practical time frame. The utility of the proposed system was confirmed in comments from specialists who noted that the system expressed the patientspecific cardiovascular state and that this was important to gain an understanding of the congenital heart disease condition.

The proposed method allows the expression of the congenital heart disease condition as diagnosed by specialists from echocardiographic images. This is expected to facilitate a shared understanding between specialists and other healthcare staff. The system could also be applicable when obtaining informed consent from families of the patient or in electronic patient records. We hope to further improve the proposed system such that it can be used in the clinical setting.

\section{ACKNOWLEDGEMENTS}

This research has been supported by the Grant-in-Aid for Scientific Research for Young Scientists (A) (21680044) from The Ministry of Education, Culture, Sports, Science and Technology, Japan.

\section{REFERENCES}

[1] G. Natesa. M. Pandian et al. “Dynamic three-dimentional echocardiography: methods and clinical potential", A Journal of Ultrasound and Allied Techniques, Vol. 11, No. 3, pp. 237259, 1994.

[2] K. Saini, M. L. Dewal, and M. Rohit, “Ultrasound Imaging and Image Segmentation in the area of Ultrasound: A Review", International Journal of Advanced Science and Technology, vol.24, pp.41-60, November, 2010.

[3] J. J. Banihachemi, E. Boidard, J. L. Bosson, L. Bressollette, I. Bricault, P. Cinquin, G. Ferretti, M. Marchal, T. Martinelli, A. Moreau-Gaudry, F. Pellissier, C. Roux, D. Saragaglia, P. Thorel, J. Troccaz, and A. Vilchis, “TER: A robot for remote ultrasonic examination: experimental evaluations, in telesurgery, S. Kumar, J.

[4] J. Hung, R. Lang, F. Flachskampf, S. K. Shernan, M. L. McCulloch, D. B. Adams, J. Thomas, M. Vannan, and T. Ryan, "3D Echocardiography: A Review of the Current Status and Future Directions ", Journal of the American Society of Echocardiography, Vol. 20, No. 3, pp. 213-233, 2007.

[5] A. S. Gopal, M. J. Schnellbaecher, Z. Shen, L. M. Boxt, J. Katz, and D. L. King, " Freehand three-dimensional echocardiography for determination of left ventricular volume and mass in patients with abnormal ventricles ", comparison with magnetic resonance imaging, J Am Soc Echocardiogr, Vol. 10, No. 8, pp. 853-861, 1997.

[6] J. Roelandt, A. Salustri, B. Mumm, and W. Vletter, “ Precordial threedimensional echocardiography with a rotational imaging probe: methods and initial clinical experience", Echocardiography Vol. 12, No. 3, pp. 243-252, 1995. 
[7] Y. Otsuji, M. D. Handschumacher et al, "Insights from three-dimensional echocardiography into the mechanism of functional mitral regurgitation: direct in vivo demonstration of altered leaflet tethering geometry ", Circulation, Vol. 96, No. 6, pp. 1999-2008, 1997.

[8] A. Mishra, P. K. Dutta, and M. K. Ghosh, "AGA based approach for boundary detection of left ventricle with echocadiographic image sequences", Image Vis. Comput., Vol. 21, pp. 967-976, 2003.

[9] M. Mignotte and J. Meunier, "A multiscale optimization approach for the dynamic contour-based boundary detection issue", Comput. Med. Imag. Graph., Vol. 25, No. 3, pp. 265-275, 2001.

[10] J. Y. Yan and T. Zhuang, "Applying improved fast marching method to endocardial boundary detection in echocardiographic images", Pattern Recognition. Letter, Vol. 24, No. 15, pp. 2777-2784, 2003.

[11] X. Wu, V. Luboz, K. Krissian, S, Cotin, and S, Dawson, "Segmentation and reconstruction of vascular structures for 3D real-time simulation", Medical Image Analysis, vol.15, issue 1, pp.22-34, February 2011.

[12] M. Nakao, T. Kuroda, M. Komori, H. Oyama, K. Minato and T. Takahashi, "Transferring Bioelasticity Knowledge through Haptic Interaction", IEEE Multimedia, Vol. 13, No. 3, pp.50-60, Jul. 2006.

[13] Y. Marchenko, I. Volkau, and W. L. Nowinski, “Vascular Editor: From Angiographic Images to 3D Vascular Models", Journal of Digit Imaging, vol.23, no.4, pp.386-398, August 2010. [14] Volkau I, Ng TT, Marchenko Y, Nowinski WL: On geometric modeling of the human intracranial venous system. IEEE Transactions on Medical Imaging 27(6):745-751, 2008.

[15] Volkau I, Zheng W, Baimouratov R, Aziz A, Nowinski

WL: Geometric modeling of the human normal cerebral arterial system. IEEE Transactions on Medical Imaging 24(4):529-539, 2005.

[16] Huang S, Baimouratov R, Volkau I, Nowinski WL, “IVME: A tool for editing, manipulation, quantification, and labeling of cerebrovascular models", Computerized Medical Imaging and Graphics, Vol. 30, pp. 187-195, 2006.

[17] O. Goksel and S. E. Salcudean, "Image-Based Variational Meshing", IEEE Trans. on Medical Imaging, Vol. 30, No. 1, pp. 11-21, 2011.

[18] M. Botsch and O. Sorkine, "On Linear Variational Surface Deformation Methods", IEEE Transactions on Visualization and Computer Graphics, Vol.14, No.1, pp. 213-230, 2008.

[19] O. Sorkine, Y. Lipman, D. Cohen-Or, M. Alexa, C. Rossl, and H. Seidel, "Laplacian surface editing", Proceedings of the 2004 Eurographics / ACM SIGGRAPH symposium on Geometry proceeding, pp. 175-184, 2004.

[20] A. Nealen, T. Igarashi, O. Sorkine, and M. Alexa, "FiberMesh: designing freeform surfaces with 3D curves", ACM Trans. On Graphics, Vol. 26, No. 3, pp. 41, 2007.

[21] U. Cagatay, "Dr. Thomas Willis' Famous Eponym: The Circle of Willis", Turkish Journal of Medical Sciences, Vol. 34, pp. 271-274, 2004.

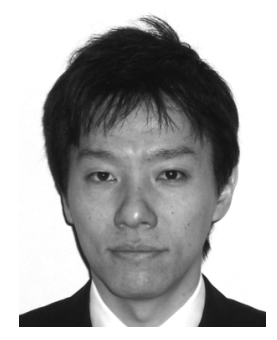

Megumi Nakao received Ph.D. degrees in informatics from Kyoto University, Kyoto, Japan, in 2003.

$\mathrm{He}$ is currently the associate professor in Biomedical Engineering Lab. at Graduate School of Informatics, Kyoto University, Japan. His research interests include biomedical graphics, computer aided surgery and medical engineering.

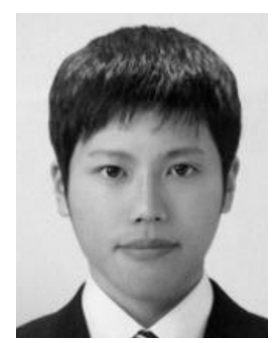

Kazuma Maeda received M.S. degrees in engineering from Nara Institute of Science and Technology (NAIST), Nara, Japan, in 2011.

He is currently working in Shimazu Corporation. He is engaged in the development of a mass spectrometer.

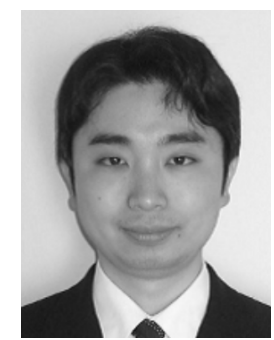

Ryo Haraguchi received Ph.D. degrees in informatics from Kyoto University, Kyoto, Japan, in 2003.

He is currently the chief in ICT Strategy Office at Research and Development Initiative Center, National Cerebral and Cardiovascular Center, Japan. His research interests include medical imaging, medical engineering and computational arrhythmology.

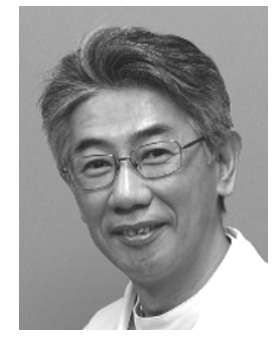

cardiology.
Ken-ichi Kurosaki received the medical degree from Kumamoto University, Kumamoto, Japan, in 1987.

$\mathrm{He}$ is currently a chief pediatric cardiac cardiologist in the section of pediatric cardiac intensive care unit, National Cerebral and Cardiovascular Center, Osaka, Japan. $\mathrm{He}$ is interested in the imaging of pediatric

Koji Kagisaki received the medical degree from Hiroshima University, Hiroshima, Japan, in 1987.

He is currently the chief in the department of pediatric cardiac surgery, National Cerebral and Cardiovascular Center, Osaka, Japan. His research interests include pediatric cardiac surgery. 


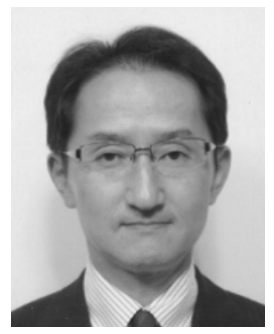

Isao Shiraishi received MD. and Ph.D. degrees in medical science from Kyoto Prefectural University of Medicine, Kyoto, Japan in 1982 and 1994, respectively.

He is currently the director of Department of Perinatal and Pediatric Cardiology, National Cerebral and Cardiovascular Center, Osaka, Japan. His research interests include 3D diagnosis of congenital heart disease using MSCT, embryonic development of the heart, and cardiovascular regeneration medicine.

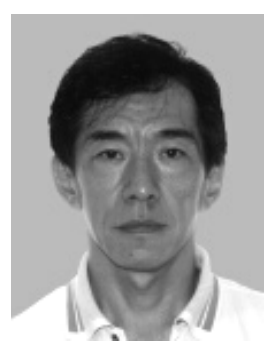

Kazuo Nakazawa received the B.S., the M.S., and the Ph.D. degrees in engineering from Osaka University, Osaka, Japan, in 1980, 1987, and 2000, respectively.

He is currently the laboratory chief in Laboratory of Biomedical Sciences and Information Management at National Cerebral and Cardiovascular center Research Institute, Japan. His research interests are medical informatics and bio-medical engineering.

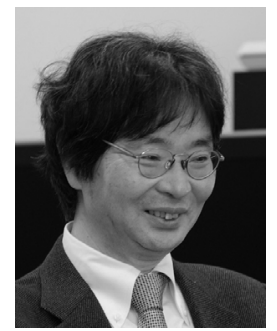

Kotaro Minato received Ph.D. degrees in electrical engineering from Kyoto University, Kyoto Japan, in 1980.

$\mathrm{He}$ is currently the dean of Graduate School of Information Science and the professor in Biomedical Imaging and Informatics Lab, Nara Institute of Science and Technology (NAIST), Japan. His research interests include medical imaging, biomedical engineering and medical informatics. 